\title{
COMPLETE RECURSIVELY ENUMERABLE SETS
}

\section{A. H. LACHLAN}

The main purpose of this paper is to improve upon the main theorem of Martin [2]. Martin gave a sufficient condition for a recursively enumerable (r.e.) set to be complete. By a slight modification we weaken Martin's condition so that it becomes both necessary and sufficient. Next we indicate briefly why Martin's condition for completeness is not a necessary one. Finally, we discuss applications of our theorem and the problem of formulating a notion of effectively maximal set. I am grateful for the referee's suggestions, particularly with regard to the statement of the theorem.

Let $W_{0}, W_{1}, \cdots$ be a standard enumeration of all r.e. sets. Let $\beta_{0}, \beta_{1}, \cdots$ be a standard enumeration of all partial recursive functions of one argument. The representing function $\kappa^{A}$ of a set of natural numbers $A$ is defined by: $\kappa^{A}(x)=0$ if $x$ is in $A, \kappa^{A}(x)=1$ if $x$ is in $\bar{A}$ (the complement of $A$ ). Let $L(x)$ denote the set of natural numbers $\leq x$.

ThEOREM. An r.e. set $C$ is complete if and only if there is a function $\tau$ recursive in $C$ and a recursive function $\phi$ such that if $\beta_{e}(z)=\kappa^{c}(z)$ for all $z \leq \tau(x)$, then $W_{\phi(e, x)} \neq W_{x}$.

Proof. For the 'if' part we adapt Martin's proof as follows. Let $C$ be an r.e. set, $\tau$ be a function recursive in $C$, and $\phi$ be a recursive function satisfying the condition of the theorem. Let $\gamma$ be a recursive function whose range is $C$, and let $C^{s}=\{\gamma(0), \gamma(1), \cdots, \gamma(s)\}$.

There is a recursive function $\pi(e, t)$ such that $\beta_{\pi(e, t)}$ is the representing function of $C^{\beta_{e}(t)}$, if $\beta_{e}(t)$ is defined, and is the empty partial function otherwise. By the recursion theorem there is a recursive function $\rho$ such that $W_{\phi(\pi(e, t), \rho(e, t))}=W_{\rho(e, t)}$ for all $e, t$. There is a function $v$ recursive in $C$ such that for all $e, t$,

$$
v(e, t)=\mu s\left[C^{s} \cap L(\tau \rho(e, t))=C \cap L(\tau \rho(e, t))\right],
$$

because the r.h.s. is effectively computable assuming a knowledge of the membership of $C$.

Let $W$ be any r.e. set. Let $\psi$ be a recursive function such that

$$
(x)[x \in W \leftrightarrow(E s)[\psi(s, x)=0]] .
$$

Let $e$ be the index of the partial recursive function $\mu s[\psi(s, x)=0]$. We shall show that $\beta_{e}(t)<v(e, t)$ for each $t$ such that $\beta_{e}(t)$ is defined. It follows at once that

Received by the editors October 15, 1966. 


$$
(x)[x \in W \leftrightarrow(E s)[s<v(e, x) \& \psi(s, x)=0]] ;
$$

hence $W$ is recursive in $C$ and $C$ is complete. Suppose then that for some $t, \beta_{e}(t)$ is defined and $\geq v(e, t)$, then

$$
C^{\beta_{\theta}(t)} \cap L(\tau \rho(e, t))=C \cap L(\tau \rho(e, t)) .
$$

Therefore $\beta_{\pi(e, t)}(z)=\kappa^{C}(z)$ for all $z \leq \tau \rho(e, t)$, whence $W_{\phi(\pi(e, t), \rho(e, t))}$ $\neq W_{\rho(\theta, t)}$. This contradicts the choice of $\rho$ and so the completeness of $C$ is established.

For the 'only if' part suppose $C$ is complete, then the r.e. sets $W_{x}$ are uniformly recursive in $C$, i.e., there exists a partial recursive functional $\Phi$ such that

$$
(x)(y)\left[y \in W_{x} \leftrightarrow \Phi\left(\kappa^{c} ; x, y\right)=0 . \& . y \notin W_{x} \leftrightarrow \Phi\left(\kappa^{c} ; x, y\right)=1\right] .
$$

Assuming knowledge of the membership of $C$ for each $x$ we can effectively compute $\tau(x)$ such that, if $\kappa^{C}(z)=\beta(z)$ for all $z \leq \tau(x)$, then $\Phi\left(\kappa^{C} ; x, y\right)=\Phi(\beta ; x, 0)$ where $\beta$ is any unary partial function. Enumerate $V_{e, x}$ as follows: given $e, x$ start computing $\Phi\left(\beta_{e} ; x, 0\right)$, if the value 1 is found enumerate 0 in $V_{e, x}$, otherwise leave $V_{e, x}$ empty. Now $V_{e, x}$ is clearly r.e. uniformly in $e, x$ and so there is a recursive function $\phi$ such that $V_{e, x}=W_{\phi(e, x)}$. Suppose that $\beta_{e}(z)=\kappa^{C}(z)$ for all $z \leq \tau(x)$ then by choice of $\tau, \Phi\left(\beta_{e} ; x, 0\right)=\Phi\left(\kappa^{c} ; x, 0\right)$, whence

$$
0 \in W_{\phi(e, x)} \leftrightarrow 0 \in V_{e, x} \leftrightarrow \Phi\left(\kappa^{c} ; x, 0\right)=1 \leftrightarrow 0 \notin W_{x} .
$$

It follows that $W_{\phi(e, x)} \neq W_{x}$ and so $C$ satisfies the condition of the theorem.

We get Martin's condition for completeness from the 'if' part of the theorem by taking $\tau(x)$ of the form $\bar{\gamma} \sigma(x)$ where $\sigma$ is a recursive function and where $\{\bar{\gamma}(0), \bar{\gamma}(1), \ldots\}$ is an enumeration of $\bar{C}$ in increasing order of magnitude.

Martin's condition for completeness is not necessary. To see this let $\gamma$ be a recursive function whose range $C$ satisfies Martin's condition. Let $W$ be any r.e. set and $\psi$ be a recursive function such that (1) holds. From [2] we see immediately that there is a recursive function $\nu$ such that for all $x$ we have

(2) $x \in W \leftrightarrow(E s)\left[\psi(x, s)=0 \& s \leqq \mu t\left[\kappa^{C}, \kappa^{C t}\right.\right.$ agree on arguments up to $\bar{\gamma} \nu(x)]]$.

Let $K$ be any fixed complete r.e. set. We now show how to simultaneously enumerate the graphs of $\gamma, \psi$, and how to effectively construct an algorithm reducing $K$ to $C$ so that there is no recursive function $\nu$ satisfying (2). The construction consists of steps $0,1, \cdots$ At each 
even step we enumerate a member of $K$ in such a way that every member of $K$ is enumerated at some step. At each odd step we 'attack' a number $e$ with a view to preventing $\nu=\beta_{e}$ from satisfying (2), in such a way that each $e$ is attacked at an infinite number of steps. We require a sequence $\left\{R_{e}\right\}$ of disjoint infinite sets which are uniformly recursive. Also, let $\left\{\beta_{e, i}\right\}$ be a strongly r.e. sequence of finite functions such that $\beta_{e, i} \subseteq \beta_{e, i+1}$ for all $e, i$, and such that $\lim _{x} \beta_{e, x}=\beta_{e}$ for all $e$. Define $W^{s}=\{x \mid \psi(x, s)=0\}$. We say that $e$ is satisfied at step $s$ if there exists $x$ in $R_{e} \cap W^{s}$ such that $\beta_{e, s}(x)$ is defined, $\bar{C}^{s}$ contains $>\beta_{e}(x)$ members $\leq \lambda(e, s)$, and $\lambda(e, s)=\lambda(e, t)$ where $t$ is the least number such that $\psi(x, t+1)=0$. To start the construction set $\gamma(0)=0, \lambda(x, 0)=x+1$ and $\psi(x, 0)=1$ for all $x$. Before any step $s$ we shall have defined $\gamma(t), \lambda(x, t)$, and $\psi(x, t)$ for all $t \leq s$ and all $x$. The general step is now described:

Step $s$ (s even). Suppose that $k$ is enumerated in $K$; we suppose this happens just once for each $k$. Define $\gamma(s+1)=\lambda(k, s), \psi(x, s+1)$ $=\psi(x, s)$ for all $x, \lambda(x, s+1)=\lambda(x, s)$ for $x<k$, and $\lambda(x, s+1)=\lambda(x+1, s)$ for $x \geq k$.

Step $s$ ( $s$ odd). Let $e$ be the number which is attacked. If $e$ is satisfied or if there exists no $x$ in $R_{e}$ such that $\beta_{e, s}(x)$ is defined and $x \notin W^{s}$, then define $\gamma(s+1)=\gamma(s), \psi(x, s+1)=\psi(x, s)$ and $\lambda(x, s+1)=\lambda(x, s)$ for all $x$. Otherwise, let $p$ be the least number in $R_{e}$ such that $\beta_{e, s}(p)$ is defined and $p \notin W^{s}$. If $\overline{C^{s}}$ contains $>\beta_{e}(p)$ members $\leq \lambda(e, s)$, define $\gamma(s+1)=\gamma(s), \psi(p, s+1)=0, \psi(x, s+1)=\psi(x, s)$ for all $x \neq p$, and $\lambda(x, s+1)=\lambda(x, s)$ for all $x$. Otherwise, set $\gamma(s+1)=\lambda(e, s), \psi(x, s+1)$ $=\psi(x, s)$ for all $x, \lambda(x, s+1)=\lambda(x, s)$ for $x<e$, and $\lambda(x, s+1)$ $=\lambda\left(x+\beta_{e}(p)+1, s\right)$ for $x \geq e$.

This completes the construction; $C$ is the range of $\gamma$ and $W$ is defined from $\psi$ by (1). We now indicate briefly why $C$ is complete and why there is no recursive $\nu$ satisfying (2). The first thing to notice is that for each $x, \lambda(x, s)$ is increasing with $s$, and that for each $s$ the sequence $\lambda(0, s), \lambda(1, s), \cdots$ is strictly increasing and contains no member of $C^{s}$. Let $P(e)$ be the proposition: at all sufficiently large steps $s$ at which $e$ is attacked, $e$ is satisfied or there exists no $x$ in $R_{e}$ such that $\beta_{e, s}(x)$ is defined and $x \notin W^{s}$. Let $Q(e)$ be the proposition: $\lim _{x} \lambda(e, x)$ exists, i.e., is finite. We prove $P(0), Q(0), P(1), Q(1), \ldots$ in that order. From $P(e)$ it follows that $\beta_{e}$ is not a recursive function such that $\nu=\beta_{e}$ satisfies (2). Since $Q(e)$ holds, knowing the membership of $C$ we can compute $s$ such that $C^{s} \cap L(\lambda(e, s))=C \cap L(\lambda(e, s))$, then $e$ cannot be enumerated in $K$ at a step $\geq s$. Thus $C$ is complete.

Our theorem can be used to weaken several well known sufficient conditions for the completeness of an r.e. set. Thus call a set $C$ reakly 
creative if it is r.e. and there is a function $\phi$ recursive in $C$ such that for all $x, W_{x} \subseteq \bar{C}$ implies $\phi(x) \notin W_{x} \cup C$. It is an easy application of the theorem to show that any weakly creative set is complete, conversely it is clear that any complete set is weakly creative. Similarly, call an r.e. set $S$ weakly effectively simple if $\bar{S}$ is infinite and there is a function $\phi$ recursive in $S$ such that for all $e, W_{e} \subseteq \bar{S}$ implies that $\phi(e)$ is greater than the cardinality of $W_{e}$. By an application of our theorem every weakly effectively simple set is complete. Also, any complete simple set is weakly effectively simple.

By analogy with the definition of 'effectively simple' it would be natural to call $M$ effectively maximal if $M$ were r.e., $\bar{M}$ infinite, and there existed a recursive function $\phi$ such that for all $e$

$$
\left[\bar{M} \cap \bar{W}_{e} \text { infinite }\right] \rightarrow\left[\phi(e)>\text { cardinality } \bar{M} \cap W_{e}\right] .
$$

Alternatively, instead of (3) we might have

$$
\left[\bar{M} \cap W_{e} \text { infinite }\right] \rightarrow\left[\phi(e)>\text { cardinality } \bar{M}-W_{e}\right] .
$$

Neither of these suggestions will do, because no coinfinite r.e. set $M$ can have either property. We would propose the following definition. Let $\kappa_{e}$ be the representing function of $W_{e}$, and let $m_{0}, m_{1}, \cdots$ be an enumeration of $\bar{M}$ in increasing order. Call an r.e. set $M$ effectively maximal if $\bar{M}$ is infinite and there exists a recursive function $\phi$ such that for all $e$ the number of alternations in the sequence $\kappa_{e}\left(m_{0}\right)$, $\kappa_{e}\left(m_{1}\right), \cdots$ is $\langle\phi(e)$. Then every effectively maximal set is clearly maximal. Also, by an application of our theorem or Martin's any effectively maximal set is complete; one should note that in this particular case use of the recursion theorem can be avoided. By examining Friedberg's construction it can be shown that the maximal set of [1] is effectively maximal provided that the enumeration $\left\{R_{i}\right\}$ of the r.e. sets on which it is based is standard.

\section{REFERENCES}

1. R. M. Friedberg, Three theorems on recursive enumeration, J. Symbolic Logic 23 (1958), 309-316.

2. D. A. Martin, Completeness, the recursion theorem, and effectively simple sets, Proc. Amer. Math. Soc. 17 (1966), 838-842.

3. J. Myhill, Creative sets, Z. Math. Logik Grundlagen Math. 1 (1955), 97-108.

4. R. M. Smullyan, Effectively simple sets, Proc. Amer. Math. Soc. 15 (1964), $893-894$.

Simon Fraser UNIVERSITY 